\title{
Solvation and Stabilization of Single Strand RNA at the Air/lce Interface Support a Primordial RNA World on Ice
}

\section{Supporting Information}

Ivan Gladich ${ }^{\dagger, \otimes, \S, *}$, Margaret L. Berrens ${ }^{x}$, Penny M. Rowe ${ }^{\ddagger}$, Rodolfo G. Pereyra ${ }^{ \pm}$, and Steven Neshyba ${ }^{\mathrm{X}, *}$

†International School for Advanced Studies (SISSA), Via Bonomea 265, 34136, Trieste, Italy

${ }^{\otimes}$ Present Affiliation: Qatar Environment and Energy Research Institute, Hamad Bin Khalifa University, P.O. Box 31110, Doha, Qatar

$\S$ European Centre for Living Technology (ECLT), Ca' Bottacin, Calle Crosera, 30124, Venice, Italy

‡NorthWest Research Associates, Redomond, WA, USA

${ }^{ \pm}$Facultad de Matemática, Astronomía, Física y Computación, Universidad

Nacional de Córdoba, X5000HUA Córdoba, Argentina

XDepartment of Chemistry, University of Puget Sound, 1500 N. Prospect St., Tacoma, Washington, USA

Contact information:

*Ivan Gladich: igladich@hbku.edu.qa

Phone number: +974 44542651

*Steven Neshyba: nesh@pugetsound.edu

Phone number: +1 253-879-3379

\section{Melting Temperature of TIP4P-D model}

Recently, the Shaw et al. have presented a new force field ${ }^{1,2}$ for nucleic acids and proteins working in combination with a new water model called TIP4P-D. ${ }^{3}$ This new force field, developed to avoid the tendency of available force fields to give disordered-state proteins that are structurally too compact relative to experiments, ${ }^{3}$ has been designed to work at room and physiological temperature. However, the adopted water model, TIP4P-D, shares stringent similarities with the TIP4P/20054 water model, which is one of the most commonly (and successfully) used water models for ice and supercooled liquid water. TIP4P-D retains the TIP4P/2005 geometry with slightly modifications of the nonbonded interaction parameters. 
TIP4P/2005 has a melting temperature of $\mathrm{T}_{\mathrm{m}}=252 \mathrm{~K}\left(\right.$ ref $\left.^{5}\right)$. It underestimates the self-diffusivity in supercooled liquid water ${ }^{6}$ but performs extremely well in terms of representing the structure of the phase diagram of water, making this water model a good candidate for interfacial phenomena at the boundaries of different phases of water. Moreover, once referenced to the same degree of supercooling ( $T$ - $T_{m}$ ) TIP4P/2005 has structural properties (e.g., ice structure, thickness of the air/ice interface, etc.) comparable to other water models for ice. TIP4P-D properties in the supercooled region have never been tested. Here, we determine the melting temperature of TIP4P-D.

The melting temperature of TIP4P-D has been determined following the same procedure in Ref. ${ }^{7}$. An initial proton disordered ice $\mathrm{Ih}$ crystal of dimension $3.2 \mathrm{~nm} \times 3.1 . \mathrm{nm} \times 5.9 \mathrm{~nm}$ for a total of 1792 water molecules was constructed using the Buch algorithm. ${ }^{8}$ This ice crystal was annealed from $0 \mathrm{~K}$ to the desired temperatures $(242 \mathrm{~K}, 244 \mathrm{~K}, 246 \mathrm{~K}, 248 \mathrm{~K}, 250 \mathrm{~K}$, and $252 \mathrm{~K})$ by performing a $1 \mathrm{~ns}$ constant pressure simulation (NpT) at 0 bar with a time step of $0.1 \mathrm{fs}$, followed by another $400 \mathrm{ps}$ at the target temperature. Afterwards, the simulation box was enlarged along the $z$ dimension to $16 \mathrm{~nm}$ giving rise to an ice slab with two vaporexposed basal ice facets, followed up by 100 ns constant volume (NVT) simulation at the target temperature. $T_{m}$ can be inferred from the evolution of total energy of the NVT runs of an ice slab with two open surfaces at different temperatures, as shown in Fig. S1. The visualization of the ice slab configuration after $100 \mathrm{~ns}$ is shown in Fig. S2. For $\mathrm{T}>\mathrm{T}_{\mathrm{m}}$ the total energy increases with time as the ice slab melts. Once the entire ice sample has melted the total energy levels off and stays constant. At $\mathrm{T}<\mathrm{Tm}$, the ice slab remains stable in time and no substantial energy increment is observed.

Figures $\mathrm{S} 1$ and $\mathrm{S} 2$ show that $\mathrm{T}_{\mathrm{m}}$ is between $248 \mathrm{~K}$ and $250 \mathrm{~K}$. Thus, we assigned $\mathrm{T}_{\mathrm{m}}=249 \mathrm{~K}$ for the TIP4P-D model. 


$$
\mathrm{T}_{\mathrm{m}}=249 \mathrm{~K}
$$

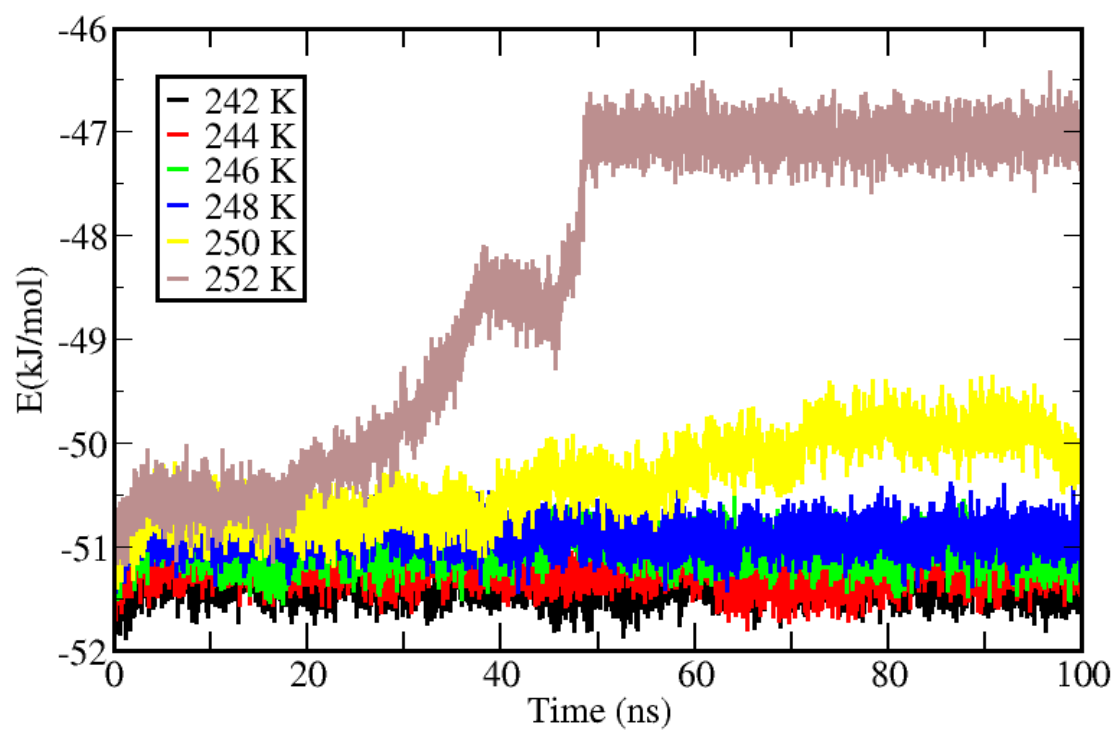

Figure S1: Total energy per molecule as a function of time in $M D$ simulations of an ice In slab at different temperatures using the TIP4P-D model. Time $t=0$ corresponds to the time when, upon equilibration of bulk ice to the target temperature, the simulation box was enlarged, and two secondary basal ice-vapor interfaces were created.

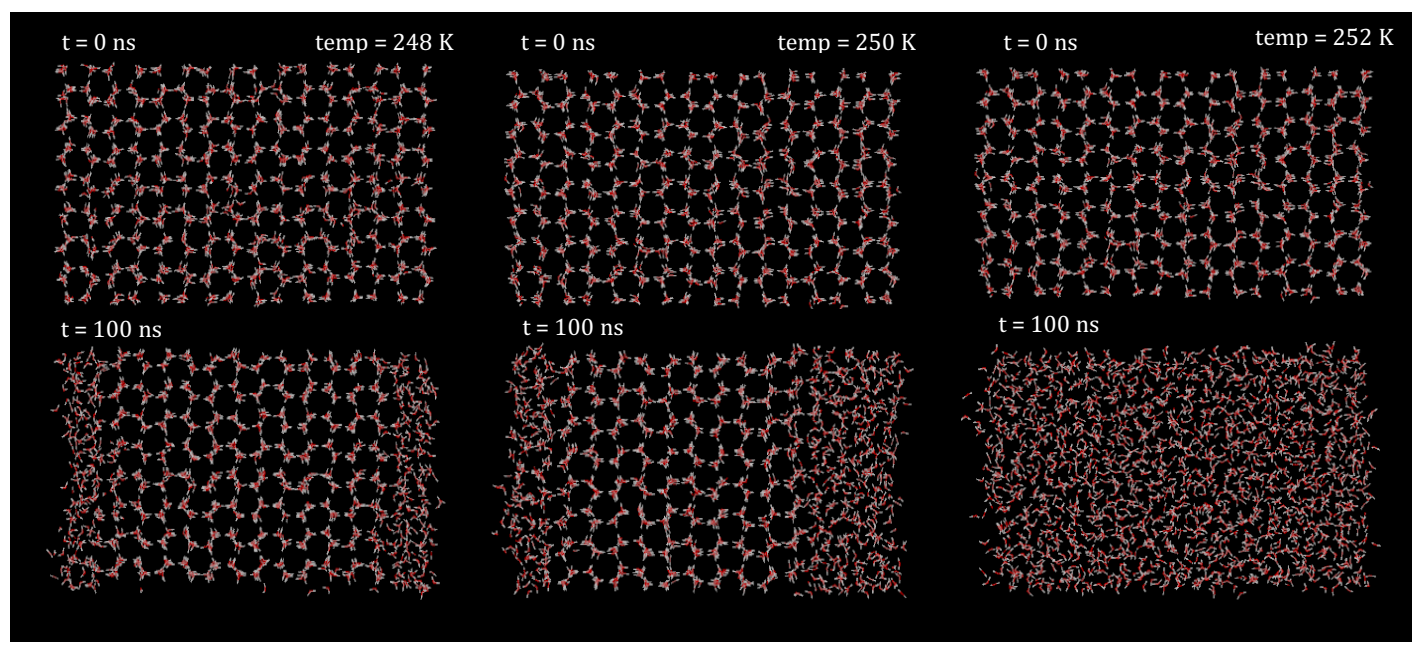

Figure S2: System configurations for the TIP4P-D ice slab at $248 \mathrm{~K}, 250 \mathrm{~K}$, and $252 \mathrm{~K}$. The top panel (a) shows the initial configurations after the equilibration run, the bottom panel (b) shows the final configurations after 100 ns simulations. 


\section{Free Energy profiles and MD simulations}

The initial configurations used for the MTD runs can be downloaded at:

https://drive.google.com/file/d/1zTC8YIXpvKZ9EhOxDI_4fAWAmRqxOiLh/view?u $\mathrm{sp}=$ sharing

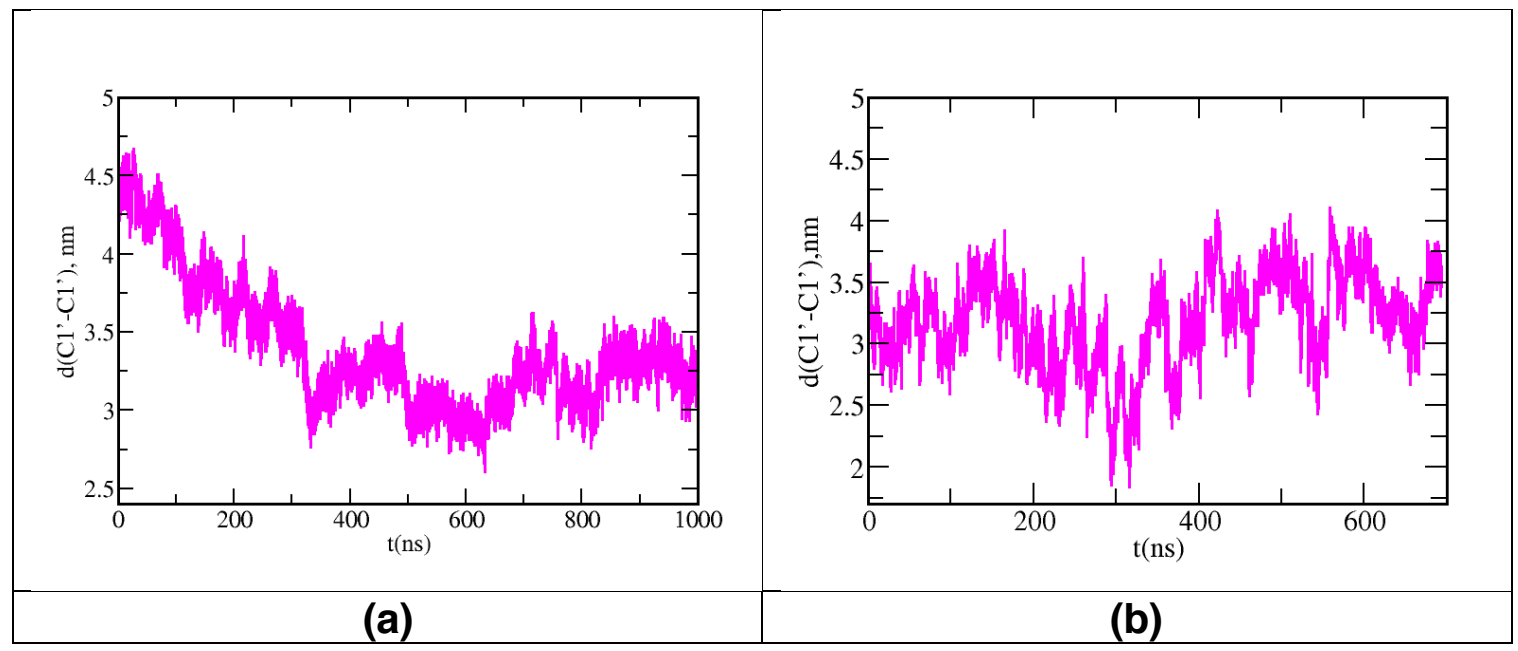

Figure S3: (a)Time series of $d\left(C 1^{\prime}-C 1\right.$ ') during the (unbiased) MD simulation of single-strand RNA on ice using as starting condition a configuration with a $\mathrm{d}_{\mathrm{C} 1^{-}-\mathrm{C}{ }^{\prime}}$ ' $\sim 4.4 \mathrm{~nm}$, i.e., slightly stretched out from the global minimum. (b)Time series of $d\left(C 1^{\prime}-C 1\right.$ ') during the (unbiased) MD simulation of single-strand RNA in bulk water at $229 \mathrm{~K}$, using as starting condition a configuration with a $d_{C 1}{ }^{\prime}-\mathrm{Cl}^{\prime} \sim 3.3 \mathrm{~nm}$. 


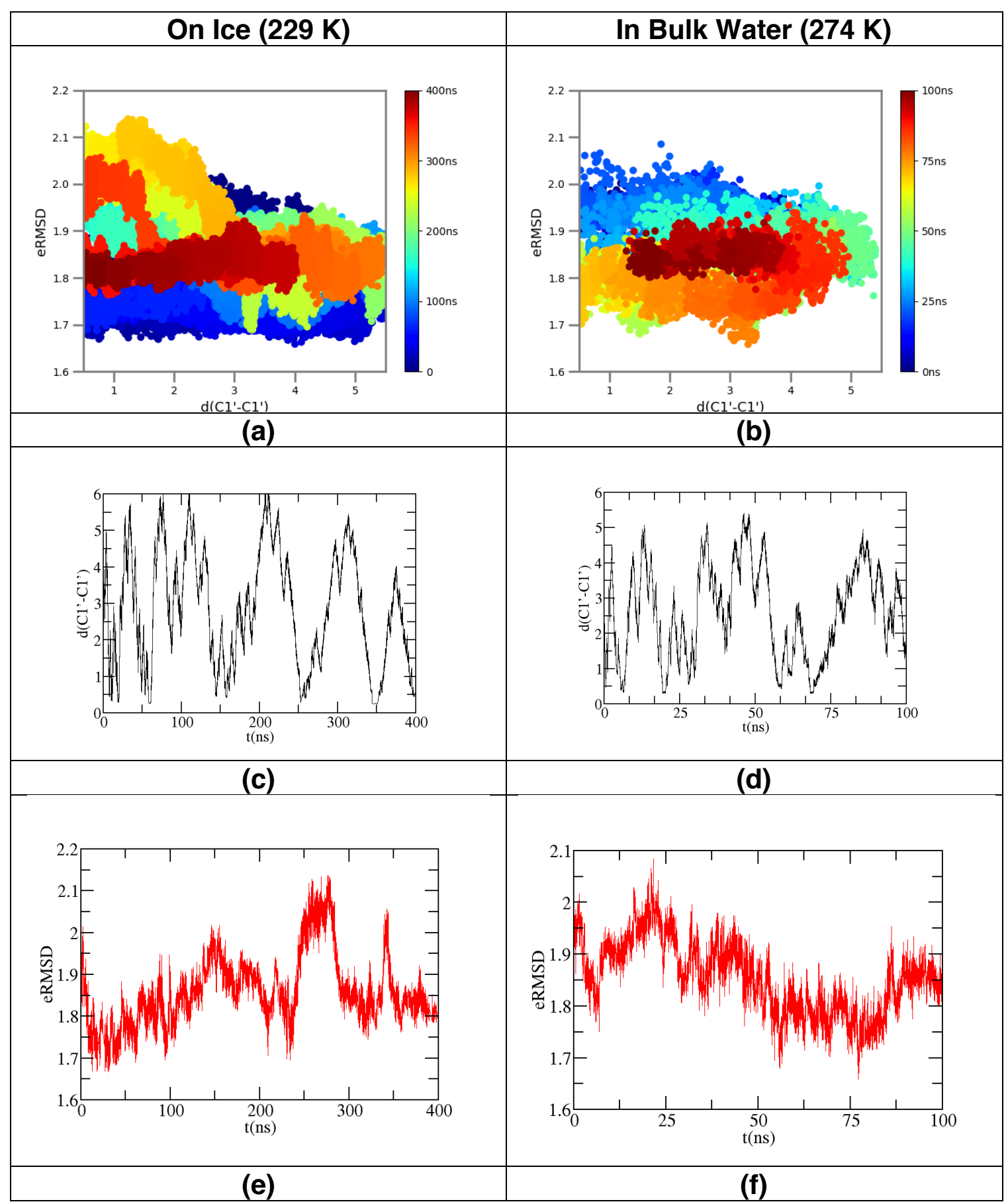

Figure S4: Panel a) and b) Scatter plots of eRMSD as a function of end-to-end C1' distance, $d\left(C 1^{\prime}-C 1^{\prime}\right)$, during the 400 ns MTD run of single-strand RNA on the basal surface of ice (a) and during the 100ns MTD run in bulk water (b). The color code corresponds to the different time spans along the MTD trajectory. Panels $c$ ) and d) show time series for the end-to-end distance during the 400 ns MTD run on ice (c) and during the 100ns MTD run in bulk water (d). Panels e) and f) show time series for the eRMSD during the 400 ns MTD run on ice (e) and during the 100ns MTD run in bulk water (f). 


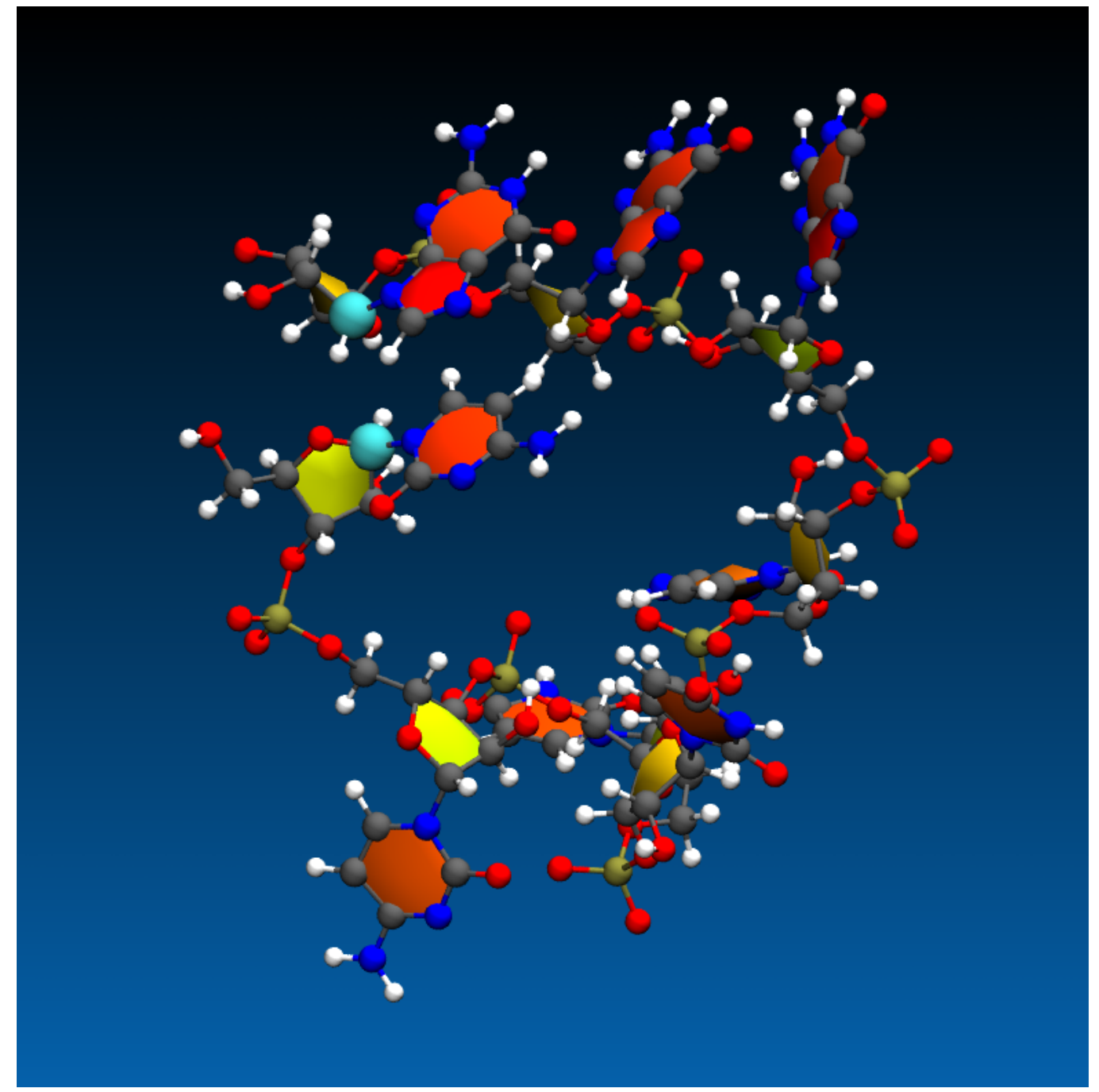

Figure S5: Snapshot take from the MTD run in bulk water at $274 \mathrm{~K}$, in which the $\mathrm{d}_{1} 1^{\prime}-\mathrm{C} 1^{\prime}<0.5 \mathrm{~nm}$. The short distance is due to the $\pi$ - stacking interaction between the two aromatic rings of the $\mathrm{C}$ and $\mathrm{G}$ (first and last) nucleotides.

\section{Supplemental References}

1. Tan, D.; Piana, S.; Dirks, R. M.; Shaw, D. E., RNA force field with accuracy comparable to state-of-the-art protein force fields. Proceedings of the National Academy of Sciences 2018.

2. $\quad$ Robustelli, P.; Piana, S.; Shaw, D. E., Developing a molecular dynamics force field for both folded and disordered protein states. Proceedings of the National Academy of Sciences 2018. 
3. Piana, S.; Donchev, A. G.; Robustelli, P.; Shaw, D. E., Water dispersion interactions strongly influence simulated structural properties of disordered protein states. J. Phys. Chem. B 2015, 119 (16), 5113-5123.

4. Abascal, J. L. F.; Vega, C., A general purpose model for the condensed phases of water: TIP4P/2005. J. Chem. Phys. 2005, 123 (23).

5. Vega, C.; Abascal, J. L. F.; Conde, M. M.; Aragones, J. L., What ice can teach us about water interactions: a critical comparison of the performance of different water models. Faraday Discuss. 2009, 141, 251-276.

6. Malaspina, D. C.; di Lorenzo, A. J. B.; Pereyra, R. G.; Szleifer, I.; Carignano, M. A., The water supercooled regime as described by four common water models. The Journal of Chemical Physics 2013, 139 (2), 024506.

7. Gladich, I.; Roeselova, M., Comparison of selected polarizable and nonpolarizable water models in molecular dynamics simulations of ice I-h. Phys. Chem. Chem. Phys. 2012, 14 (32), 11371-11385.

8. Buch, V.; Sandler, P.; Sadlej, J., Simulations of H2O solid, liquid, and clusters, with an emphasis on ferroelectric ordering transition in hexagonal ice. J. Phys. Chem. $B$ 1998, 102 (44), 8641-8653. 